\title{
Regioselective cobalt(II)-catalyzed [2 + 3] cycloaddition reaction of fluoroalkylated alkynes with 2-formylphenyl- boronic acids: easy access to 2-fluoroalkylated indenols
}

Tatsuya Kumon, Miroku Shimada, Jianyan Wu, Shigeyuki Yamada and Tsutomu Konno*

Open Access

\author{
Full Research Paper \\ Address: \\ Faculty of Molecular Chemistry and Engineering, Kyoto Institute of \\ Technology, Matsugasaki, Sakyo-ku, Kyoto 606-8585, Japan \\ Email: \\ Tsutomu Konno* - konno@kit.ac.jp \\ * Corresponding author \\ Keywords: \\ [2 + 3] cycloaddition; cobalt catalyst; fluorine-containing; indenols; \\ regioselective
}

\author{
Beilstein J. Org. Chem. 2020, 16, 2193-2200. \\ https://doi.org/10.3762/bjoc.16.184 \\ Received: 13 July 2020 \\ Accepted: 25 August 2020 \\ Published: 04 September 2020 \\ This article is part of the thematic issue "Organo-fluorine chemistry V". \\ Guest Editor: D. O'Hagan \\ (c) 2020 Kumon et al.; licensee Beilstein-Institut. \\ License and terms: see end of document.
}

\begin{abstract}
$[2+3]$ cycloaddition reactions of fluorinated alkynes with 2-formylphenylboronic acids under the influence of $\mathrm{Co}(\mathrm{acac})_{2} \cdot 2 \mathrm{H}_{2} \mathrm{O}$ in two-component solvents of acetonitrile/2-propanol at reflux temperature for $18 \mathrm{~h}$ took place smoothly, affording the corresponding fluoroalkylated indenol derivatives in good yields. This reaction shows excellent regioselectivity, giving 2-fluoroalkylated indenols, together with a very small amount of 3-fluoroalkylated indanones as side products.
\end{abstract}

\section{Introduction}

2,3-Disubstituted indenol derivatives are important compounds possessing high potential due to the insecticidal, myorelaxation, and antiproliferative properties (Figure 1) [1-12]. Thus, enormous attention has been paid to 2- or 3-fluoroalkylated indenol derivatives in the field of medicinal and agrochemical drug design since a fluorine atom can very often bring about an increasing effect on the pharmacological activity owing to the unique nature of the fluorine atom(s) [13-18]. However, reports on a synthetic protocol for 2- or 3-fluoroalkylated indenols are very limited [19-22]. Recently, Yamazaki et al. have reported the reaction of the $\mathrm{CF}_{3}$-containing phthalide $\mathbf{B}$ prepared from the 1,2-diester $\mathbf{A}$ with the Ruppert-Prakash reagent ( $\mathrm{TMSCF}_{3}$ ) in the presence of a catalytic amount of $\mathrm{CsF}$ (Scheme 1a). Ac- cording to this paper, phthalide $\mathbf{B}$ reacted smoothly with phenylthiomethyllithium to give the corresponding $\mathrm{CF}_{3}-$ containing indanone $\mathbf{C}$. Then, dehydration of $\mathbf{C}$ using $p$ - $\mathrm{TsOH} \cdot \mathrm{H}_{2} \mathrm{O}$, followed by a 1,2-reduction led to 3-fluoroalky-<smiles>[R]C1=C(O)c2ccccc2C1[R]</smiles>

Figure 1: Indenol skeleton. 
synthesis of 3-fluoroalkylated indenol

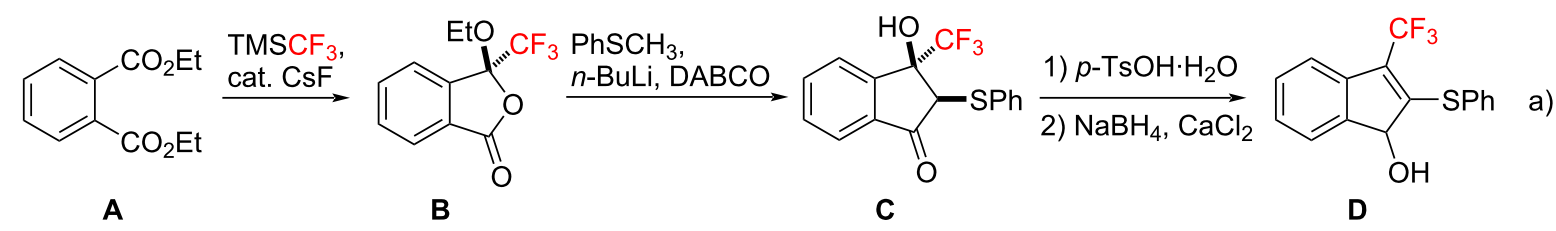

transition-metal-catalyzed [2+3] cycloaddition

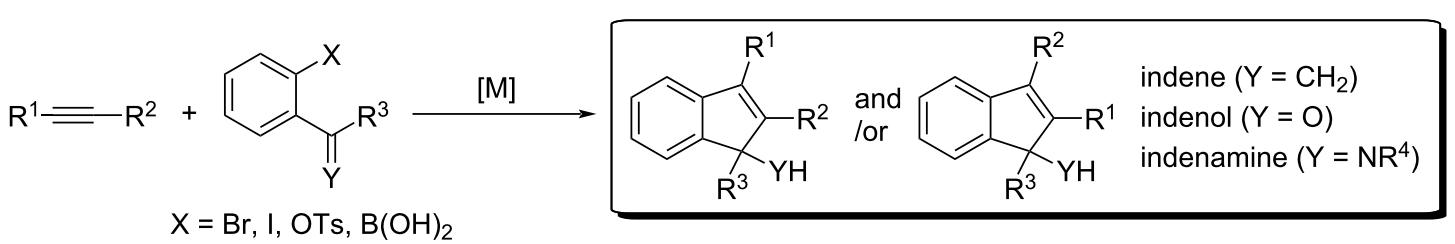

our previous work

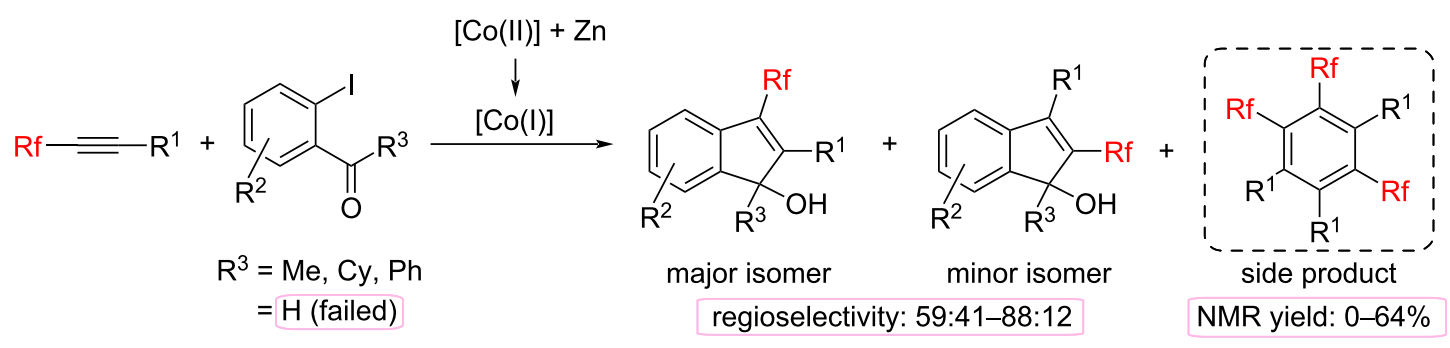

this work

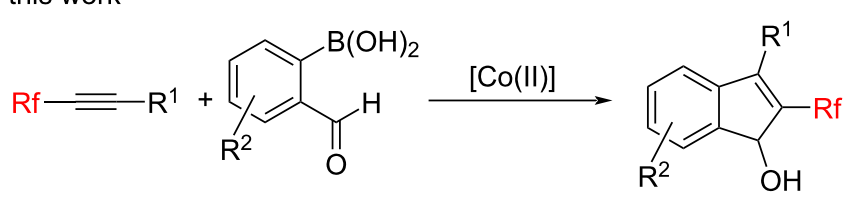<smiles>[R]C1C(=O)c2ccccc2C1[R]</smiles>

lated indenol D. All of these reactions produced the desired products in good yield, however, this protocol is limited to the introduction of a trifluoromethyl group at the 3-position of the indenol. To the best of our knowledge, there are no reports on the practical synthesis of disubstituted 2-fluoroalkylated indenols so far.

Transition-metal-catalyzed carbocyclization reactions of alkynes with benzene derivatives having a leaving group $\mathrm{X}$ ( $\mathrm{X}=\mathrm{Br}$, I, OTs, $\mathrm{B}(\mathrm{OH})_{2}$ ) have been widely considered as one of the most efficient and convenient protocols for the construction of various 2,3-disubstituted indene derivatives, such as indenols and indenamines (Scheme 1b) [23-25]. There have been numerous studies on the reaction with nonfluorinated alkynes under the influence of various transition metals. Despite their effective advantages, on the other hand, the reports on cycloaddition reactions with fluorine-containing alkynes for the construction of fluoroalkylated indene derivatives are rare $[21,26]$.

Recently, our group has reported the first practical synthesis of fluoroalkylated indenol derivatives using various fluoroalkylated alkynes with 2-iodoaryl ketones via cobalt-catalyzed carbocyclizations (Scheme 1c) [21]. Although our previous work was practical to produce interesting fluoroalkylated indenol derivatives, some drawbacks still remain unsolved. Initially, the reaction showed a low regioselectivity, leading to a mixture of 3-fluoroalkylated and 2-fluoroalkylated indenols, which was difficult to separate. Secondly, a trimer of fluoroalkylated alkynes was obtained as a side product since the cobalt(I) species produced by the cobalt(II)/Zn system worked as a suitable catalyst, leading to the corresponding trimer of the 
fluoroalkylated alkyne, as reported by our group [27]. Finally, only 2-iodoaryl ketones $\left(\mathrm{R}^{3}=\mathrm{Me}, \mathrm{Cy}, \mathrm{Ph}\right)$ were applicable in this catalytic reaction, whereas the cycloaddition using 2-iodobenzaldehyde $\left(\mathrm{R}^{3}=\mathrm{H}\right)$ did not work at all. Therefore, the development of practical protocols for [2 +3$]$ cycloaddition reactions with a broader substrate scope for the synthesis of fluoroalkylated indenols is still required. Herein we present a synthesis of 2-fluoroalkylated indenols via $[2+3]$ cycloadditions of various fluorinated alkynes with 2-formylphenylboronic acids in the presence of cobalt(II) species as a catalyst to suppress the trimerization products (Scheme 1d).

\section{Results and Discussion}

Initially, we carried out the screening of the reaction conditions for the cobalt-catalyzed [2+3] cycloaddition using fluoroalkylated alkyne 1a and 2-formylphenylboronic acid (2A) [28]. The results are summarized in Table 1.

The cycloaddition of the fluoroalkylated alkyne 1a with 2.0 equiv of 2-formylphenylboronic acid $(\mathbf{2 A})$ in the presence of $10 \mathrm{~mol} \%$ each of $\mathrm{Co}(\mathrm{acac})_{2} \cdot 2 \mathrm{H}_{2} \mathrm{O}$ and 1,2 - bis(diphenylphosphino)ethane (dppe) in $\mathrm{CH}_{3} \mathrm{CN}$ at $80{ }^{\circ} \mathrm{C}$ for $18 \mathrm{~h}$ proceeded to afford the corresponding cyclic products $\mathbf{3 a A}$ and $\mathbf{4 a A}$ in $76 \%$ yield as a regioisomeric 66:34 mixture (Table 1, entry 1).
Intriguingly, a small amount of the undesired trans-3-fluoroalkylated indanone 5aA was obtained as a side product, whereas the cis-3-fluoroalkylated and cis/trans-2-fluoroalkylated indanones were not observed. As shown in Table 1, entries 2-4, changing the solvent from $\mathrm{CH}_{3} \mathrm{CN}$ to DCE, iPrOH, or 1,4dioxane caused an appreciable decrease in the yield. It should be noted that the use of a mixed solvent of $\mathrm{CH}_{3} \mathrm{CN}$ and $\mathrm{iPrOH}$ significantly improved the isomeric ratio of $\mathbf{3 a A}$ and $4 \mathbf{a A}$ (>99:1), although $48 \%$ of the indanone $\mathbf{5 a A}$ was also obtained (Table 1, entry 5). The cycloaddition reaction in the presence of $\mathrm{CoCl}_{2}$ instead of $\mathrm{Co}(\mathrm{acac})_{2} \cdot 2 \mathrm{H}_{2} \mathrm{O}$ produced the desired $\mathbf{3 a A}$ and 4aA in $81 \%$ yield, with a lower selectivity of 66:34 (Table 1, entry 6), while the other cobalt catalysts, such as $\mathrm{Co}(\mathrm{OAc})_{2} \cdot 4 \mathrm{H}_{2} \mathrm{O}, \mathrm{Co}(\mathrm{acac})_{3}$, and $\mathrm{Co}(\mathrm{OH})_{2}$ resulted in the sluggish formation of the fluoroalkylated indenols or indanone (Table 1, entries 7-9). When 1,3-bis(diphenylphosphino)propane (dppp) was used as a phosphine ligand, the desired indenol 3aA was obtained in a moderate yield and with high regioselectivity of 98:2, together with a very small amount of the fluoroalkylated indanone 5aA (Table 1, entry 10). On the other hand, 1,4-bis(diphenylphosphino)butane (dppb) was not a suitable ligand for this reaction, leading only to trace amount of the indanone 5aA (Table 1, entry 11). Finally, the desired cyclization product was produced in a good yield at the reflux tem-

Table 1: Screening for the reaction conditions of the cobalt-catalyzed [2 +3$]$ cycloaddition using the fluoroalkylated alkyne 1a and 2 -formylphenylboronic acid (2A)

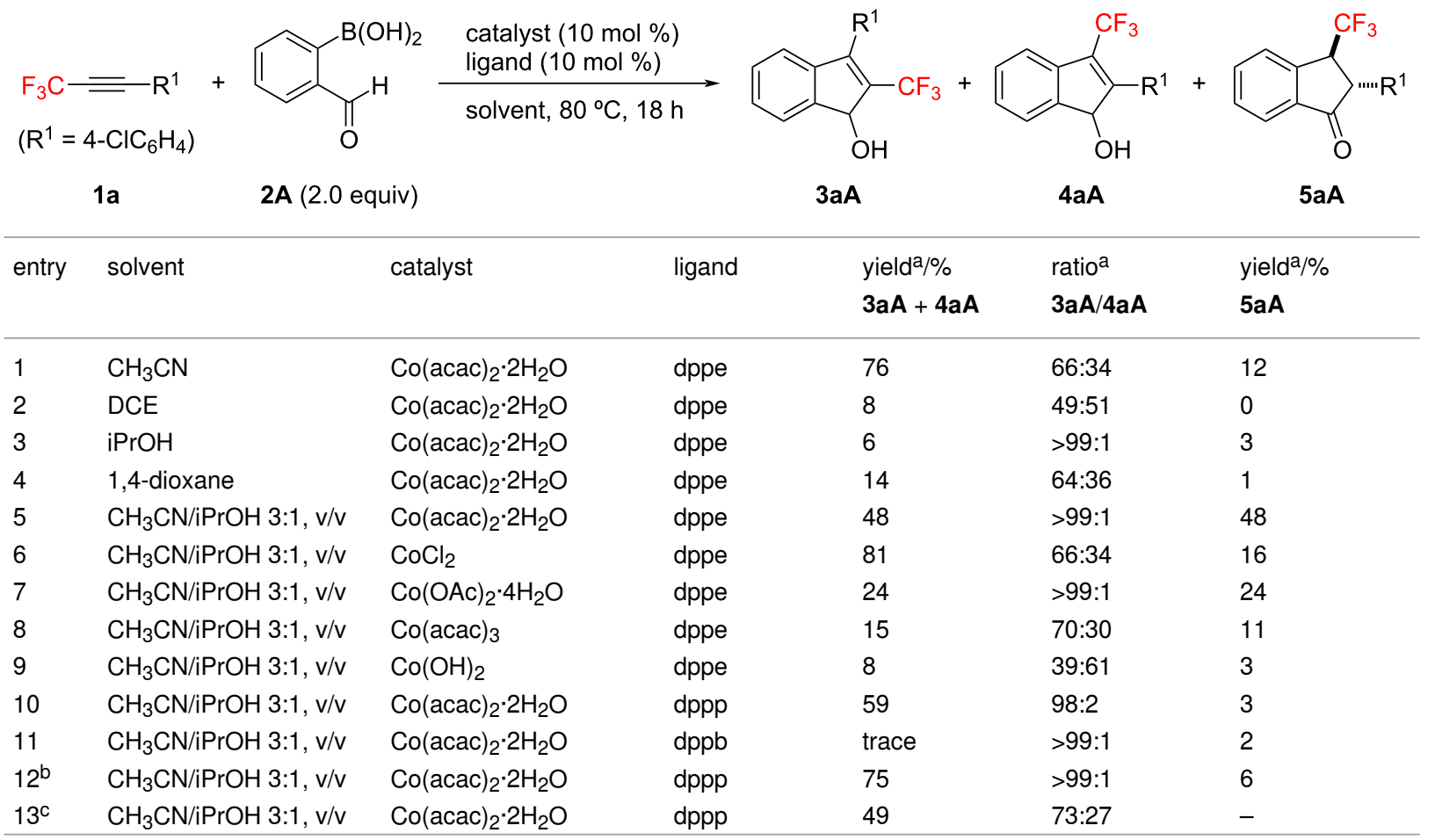

aDetermined by ${ }^{19} \mathrm{~F}$ NMR spectroscopy. ${ }^{\mathrm{b}} \mathrm{Carried}$ out at the reflux temperature. ${ }^{\mathrm{c}} 2$-Acetylphenylboronic acid was used. 
perature while maintaining the selectivity ( $>99: 1$, Table 1 , entry 12). Unfortunately, 2-acetylphenylboronic acid instead of 2-formylphenylboronic acid (2A) showed a lower reactivity and a poor regioselectivity (73:27, Table 1 , entry 13) [29].
With the optimized reaction conditions (Table 1, entry 12), we explored the substrate scope for the $[2+3]$ cycloaddition of various fluoroalkylated alkynes $\mathbf{1}$ and 2-formylphenylboronic acids $\mathbf{2}$. The results are summarized in Scheme 2.

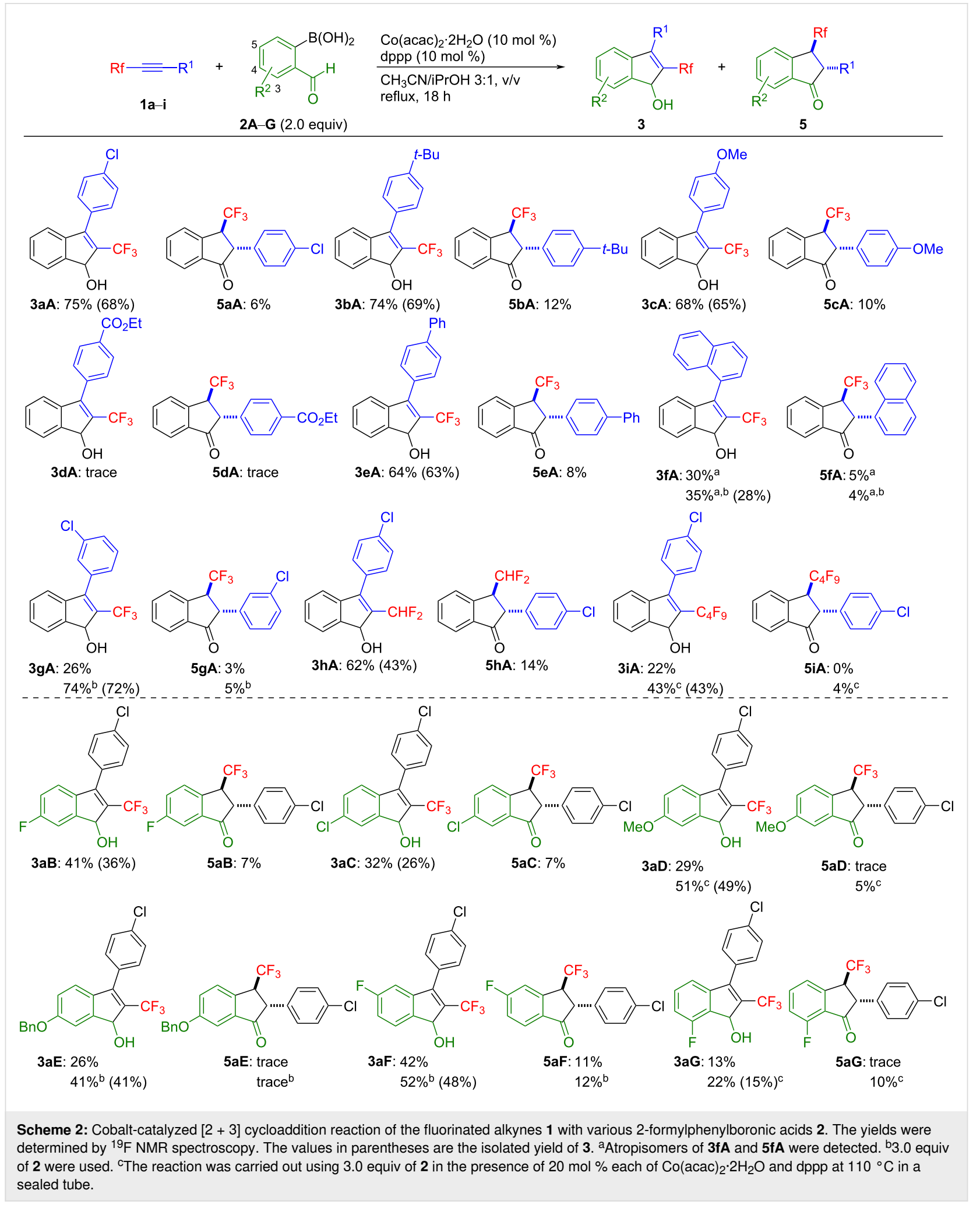


The substrates 1, having an electron-donating substituent on the benzene ring of the fluoroalkylated alkyne, such as $t$-Bu or $\mathrm{MeO}$, reacted efficiently, leading to the desired fluoroalkylated indenols $\mathbf{3 b A}$ and $\mathbf{3 c A}$ in $74 \%$ and $68 \%$ yield, respectively. However, the substrate containing the electron-withdrawing group $\mathrm{CO}_{2} \mathrm{Et}$ on the benzene ring of $\mathbf{1}$ showed a lower reactivity in this reaction (see $\mathbf{3 d A}$ ). The cycloaddition reaction using a fluoroalkylated alkyne having a 4-biphenyl group as $\mathrm{R}^{1}$ took place smoothly, giving the corresponding indenol $\mathbf{3 e A}$ in a good yield. Though bulkier groups as $\mathrm{R}^{1}$, such as 1-naphthyl or 3-chlorophenyl, reduced the reactivity (see $\mathbf{3 f A}$ and $\mathbf{3 g A}$ ), an excess loading of the boronic acid improved the yield appreciably. For the difluoromethylated alkyne $\mathbf{1 h}$, the desired indenol $3 \mathrm{hA}$ was obtained in $62 \%$ yield under optimal conditions. However, the nonafluorobutylated alkyne 1i showed a lower reactivity, giving the corresponding indenol $\mathbf{3 i A}$ in $22 \%$ yield. Therefore, when the reaction was carried out using 3.0 equiv of the boronic acid $\mathbf{2 A}$ and $20 \mathrm{~mol} \%$ each of $\mathrm{Co}(\mathrm{acac})_{2} \cdot 2 \mathrm{H}_{2} \mathrm{O}$ and dppp at $110{ }^{\circ} \mathrm{C}$, the desired 2-fluoroalkylated indenol $3 \mathbf{i A}$ was obtained in $43 \%$ yield.

Subsequently, we investigated the $[2+3]$ cycloaddition reaction of the fluoroalkylated alkyne $\mathbf{1 a}\left(\mathrm{R}^{1}=4-\mathrm{ClC}_{6} \mathrm{H}_{4}\right)$ with variously substituted 2-formylphenylboronic acids 2 . Electrondeficient formylphenylboronic acids possessing a fluorine or chlorine atom on the benzene ring gave the corresponding indenols $\mathbf{3 a B}$ and $\mathbf{3 a C}$ in $41 \%$ and $32 \%$ yield, respectively, which could not be improved even when an excess loading of the reagents, prolonging the reaction time, and a higher reaction temperature were applied. However, the exposure of the fluoroalkylated alkynes 1a to electron-rich substrates, e.g., 2D and $\mathbf{2 E}$, lead to the indenols in approximately $30 \%$ yield, and the use of an excessive amount of boronic acid, $\mathrm{Co}(\mathrm{acac})_{2} \cdot 2 \mathrm{H}_{2} \mathrm{O}$, or dppp improved the yields of the desired indenols, forming $\mathbf{3 a D}$ in $51 \%$ and $\mathbf{3 a E}$ in $41 \%$ yield. Changing the substituent position on the aromatic ring of the phenylboronic acid from the 4to the 5-position did not affect the result, giving the indenol $\mathbf{3 a F}$ in $52 \%$ yield. However, a boronic acid with a fluorine atom at the 3-position did not lead to a satisfactory result, with the cyclic product $\mathbf{3 a G}$ being formed in only $22 \%$ yield.

Substituted indenones and indanones widely exist in nature, and these skeletons are important classes of organic compounds due to diverse biological and pharmacological activities [30-32]. Therefore, we also accomplished the synthesis of 2-fluoroalkylated indenone and indanone by simple reactions (Scheme 3 ). An allylic oxidation was carried out with 10 equiv of manganese dioxide in dichloromethane as the solvent at $0{ }^{\circ} \mathrm{C}$ for 30 minutes, leading to the corresponding 2-fluoroalkylated indenone 6 in $98 \%$ yield. Subsequently, a hydrogenolysis with $1 \mathrm{~mol} \%$ of $\mathrm{Pd} / \mathrm{C}$ under a hydrogen atmosphere in methanol at room temperature for $15 \mathrm{~h}$ produced the desired 2-fluoroalkylated indanone 7 as the trans-isomer in 69\% yield [33-35].

The stereochemical assignment of $\mathbf{5 a A}$ and $\mathbf{7}$ was carried out based on NMR techniques, as shown in Scheme 4 [36]. A strong correlation between the carbonyl carbon atom and $\mathrm{H}_{\mathrm{a}}$ was obtained, whereas the cross-peak between the carbonyl carbon atom and $\mathrm{H}_{\mathrm{b}}$ was not observed, strongly indicating that the indanone 5aA possessed a $\mathrm{CF}_{3}$ group at the 3-position. On the other hand, the cross-peak between the carbonyl carbon atom and $\mathrm{H}_{\mathrm{c}}$ of the indanone 7 was obtained, but there was no crosspeak between the carbonyl carbon atom and $\mathrm{H}_{\mathrm{d}}$, meaning that the indanone 7 has a $\mathrm{CF}_{3}$ group at the 2-position. This result indicated that the indenol $\mathbf{3} \mathbf{a} \mathbf{A}$, which is the precursor of the 2-fluoroalkylated indanone 7, also possess a trifluoromethyl group at the 2-position. Each $\mathrm{H}_{\mathrm{a}}-\mathrm{H}_{\mathrm{b}}$ coupling of $\mathbf{5 a A}$ and each $\mathrm{H}_{\mathrm{c}}-\mathrm{H}_{\mathrm{d}}$ coupling of 7 appeared as a doublet, with $J=4.0 \mathrm{~Hz}$ and $4.6 \mathrm{~Hz}$, respectively. These NMR results suggested that the fluoroalkylated indanones $\mathbf{5 a A}$ and $\mathbf{7}$ were trans-isomers, and previous results showed that the $J$ value of the nonfluoroalkylated trans-2,3-disubstituted indanone 8 is $5.0 \mathrm{~Hz}$, whereas the $J$ value of the cis-isomer 9 is $8.0 \mathrm{~Hz}$ [37].

The proposed catalytic cycles for the cobalt-catalyzed [2+3] cycloaddition process leading to 2-fluoroalkylated indenols and

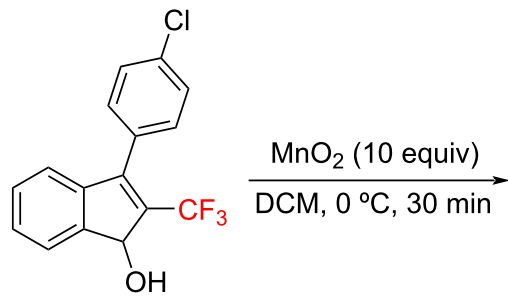

3aA

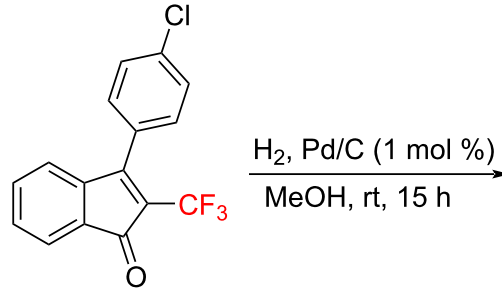<smiles>O=C1c2ccccc2C(c2ccc(Cl)cc2)C1C(F)(F)F</smiles>

7 $69 \%(58 \%)^{\mathrm{a}}$

Scheme 3: Synthesis of the fluoroalkylated indenone 6 and the indanone 7 from the indenol 3aA. The yields were determined by ${ }^{19} \mathrm{~F}$ NMR spectroscopy. The values in parentheses are the isolated yields. 


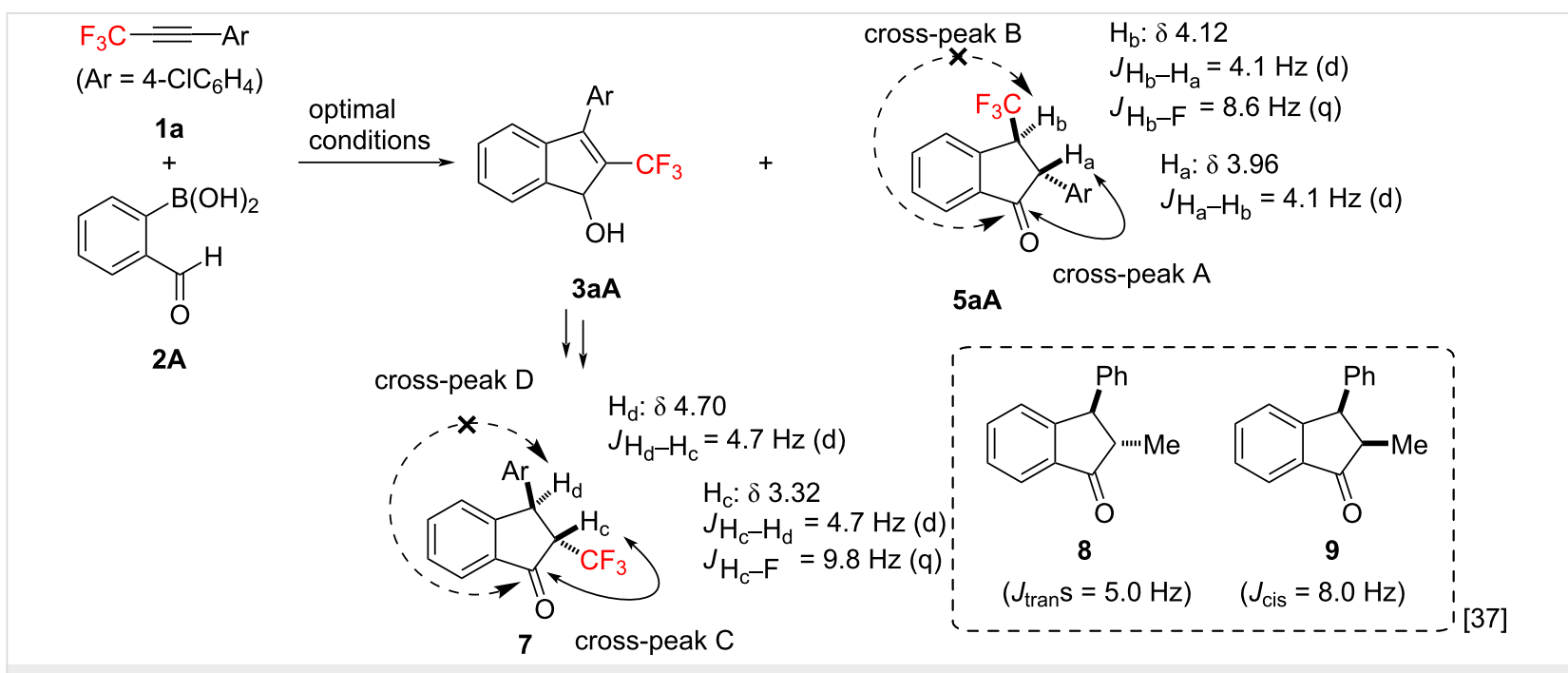

Scheme 4: Stereochemical assignment of $\mathbf{5 a A}$ and 7 based on NMR techniques. The cross-peaks were observed through HMBC measurements.

3-fluoroalkylated indanones are shown in Scheme 5 [28,38]. Thus, the reaction presumably proceeds as follows: (1) transmetalation of the cobalt catalyst with 2-formylphenylboronic acids (2) gives the arylcobalt species Int-1, (2) insertion of the alkyne 1 into the [Co]-Ar bond (see Int-2a) [39], (3) migration insertion into the formyl moiety to afford the corresponding cobalt alkoxide Int-3a, (4) protonation of Int-3a with HX (X = acac, $\mathrm{OiPr}$, or $\mathrm{OH}$ ), giving rise to the desired 2-fluoroalkylated indenol 3.

The formation of the 3-fluoroalkylated indanones as a side product may be explained based on the previous literature

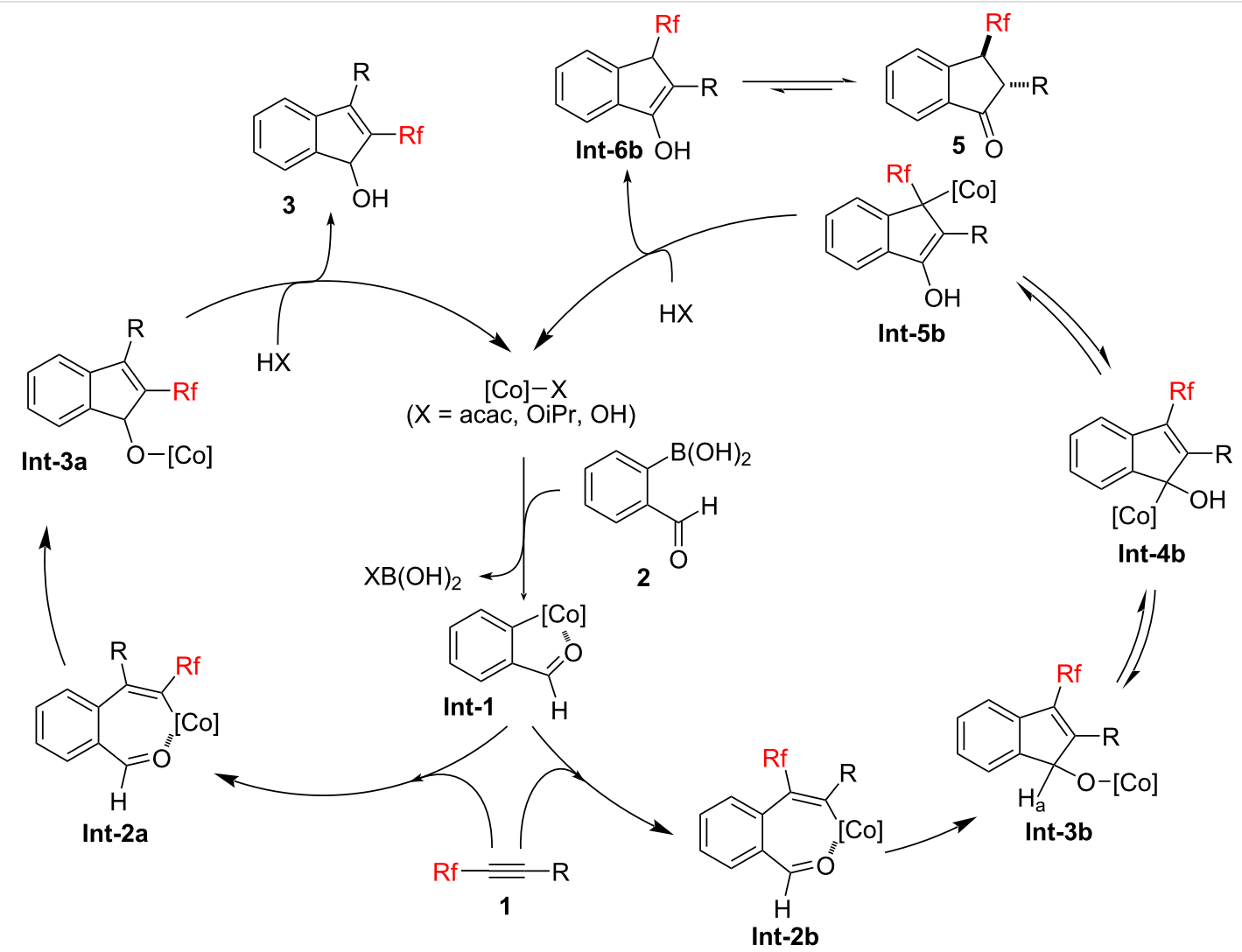

Scheme 5: Proposed reaction mechanism. 
[20,40]. The reaction of Int-1 with the alkynes $\mathbf{1}$ also gives Int-2b as a regioisomeric intermediate of Int-2a, leading to the 3-fluoroalkylated cobalt alkoxide Int-3b. Subsequently, the proton shift of the cobalt alkoxide Int-3b provides the allylcobalt species Int-4b because the acidity of the proton $\mathrm{H}_{\mathrm{a}}$ of Int-3b is the same as, or higher than the hydroxy group of Int-4b due to the electron-withdrawing effect of the fluoroalkyl group. It should be noted that this process is probably accelerated by 2-propanol or $\mathrm{H}_{2} \mathrm{O}$, which is explained by the results that the most 3-fluoroalkylated indenol $\mathbf{4 a A}$ was converted into the indanone $\mathbf{5 a A}$ in the reaction, using 2-propanol as the solvent and a cobalt hydrate as a catalyst, such as $\mathrm{Co}(\mathrm{acac})_{2} \cdot 2 \mathrm{H}_{2} \mathrm{O}$ and $\mathrm{Co}(\mathrm{OAc})_{2} \cdot 4 \mathrm{H}_{2} \mathrm{O}$, respectively (Table 1 , entries 3, 5, and 7).

The compound Int-4b produced the allylcobalt species Int-5b with a stabilized $\mathrm{C}-[\mathrm{Co}]$ bond due to the electronwithdrawing ability of the fluoroalkyl group. The Int-5b species undergoes protonation at the carbon bonded to the fluoroalkyl group, giving the enol Int-6b. Finally, the enol Int-6b produces the 3 -fluoroalkylated indanone $\mathbf{5}$ via keto-enol tautomerism.

\section{Conclusion}

In conclusion, we developed a practical and efficient synthetic protocol for 2-fluoroalkylated indenol derivatives via a cobaltcatalyzed [2 + 3] cycloaddition reaction using fluorinated alkynes and 2-formylphenylboronic acids. It was revealed that the reaction using dppp as a ligand showed a high regioselectivity, leading to 2-fluoroalkylated indenols in good yield. Moreover, the side product was a small amount of 3-fluoroalkylated indanones, which was easily separated from the 2-fluoroalkylated indenols due to the lower polarity of indanones compared to indenols. Finally, 2-fluoroalkylated indenol was simply converted into the corresponding 2-fluoroalkylated indenone and indanone in good to excellent yield.

\section{Supporting Information}

\section{Supporting Information File 1}

Experimental procedures, characterization data $\left({ }^{1} \mathrm{H},{ }^{13} \mathrm{C}\right.$, ${ }^{19} \mathrm{~F}$ NMR, IR, and HRMS), copies of ${ }^{1} \mathrm{H},{ }^{13} \mathrm{C}$, and ${ }^{19} \mathrm{~F}$ NMR spectra.

[https://www.beilstein-journals.org/bjoc/content/ supplementary/1860-5397-16-184-S1.pdf]

\section{Acknowledgements}

We sincerely thank the Tosoh Finechem Corp. for a generous gift of 2-bromo-3,3,3-trifluoroprop-1-ene.

\section{ORCID ${ }^{\circledR}$ iDs}

Tatsuya Kumon - https://orcid.org/0000-0003-0862-1218 Shigeyuki Yamada - https://orcid.org/0000-0002-6379-0447

Tsutomu Konno - https://orcid.org/0000-0002-5146-9840

\section{References}

1. Ishiguro, Y.; Okamoto, K.; Ojima, F.; Sonoda, Y. Chem. Lett. 1993, 22 , 1139-1140. doi:10.1246/cl.1993.1139

2. Clark, W. M.; Tickner-Eldridge, A. M.; Huang, G. K.; Pridgen, L. N.; Olsen, M. A.; Mills, R. J.; Lantos, I.; Baine, N. H. J. Am. Chem. Soc. 1998, 120, 4550-4551. doi:10.1021/ja973882j

3. Jiang, Z.-H.; Hwang, G.-S.; Xi, Z.; Goldberg, I. H. J. Am. Chem. Soc. 2002, 124, 3216-3217. doi:10.1021/ja0176292

4. Upadhayaya, R. S.; Shinde, P. D.; Kadam, S. A.; Bawane, A. N.; Sayyed, A. Y.; Kardile, R. A.; Gitay, P. N.; Lahore, S. V.; Dixit, S. S.; Földesi, A.; Chattopadhyaya, J. Eur. J. Med. Chem. 2011, 46, 1306-1324. doi:10.1016/j.ejmech.2011.01.053

5. Liebeskind, L. S.; Gasdaska, J. R.; McCallum, J. S.; Tremont, S. J. J. Org. Chem. 1989, 54, 669-677. doi:10.1021/jo00264a030

6. Robinson, N. P.; Main, L.; Nicholson, B. K. J. Organomet. Chem. 1989, 364, C37-C39. doi:10.1016/0022-328x(89)87157-8

7. Quan, L. G.; Gevorgyan, V.; Yamamoto, Y. J. Am. Chem. Soc. 1999, 121, 3545-3546. doi:10.1021/ja983645w

8. Chang, K.-J.; Rayabarapu, D. K.; Cheng, C.-H. J. Org. Chem. 2004, 69, 4781-4787. doi:10.1021/jo049506g

9. Singh, P. P.; Reddy, P. B.; Sawant, S. D.; Koul, S.; Taneja, S. C.; Kumar, H. M. S. Tetrahedron Lett. 2006, 47, 7241-7243. doi:10.1016/j.tetlet.2006.07.126

10. Chinnagolla, R. K.; Jeganmohan, M. Eur. J. Org. Chem. 2012, 417-423. doi:10.1002/ejoc.201101364

11. Zhang, C.; Li, H.; Pei, C.; Quu, L.; Hu, W.; Bao, X.; Xu, X. ACS Catal. 2019, 9, 2440-2447. doi:10.1021/acscatal.8b04144

12. Gu, C.-X.; Chen, W.-W.; Xu, M.-H. J. Org. Chem. 2020, 85, 3887-3893. doi:10.1021/acs.joc.9b02958

13. Meanwell, N. A. J. Med. Chem. 2018, 61, 5822-5880. doi:10.1021/acs.jmedchem.7b01788

14. Zhou, Y.; Wang, J.; Gu, Z.; Wang, S.; Zhu, W.; Aceña, J. L.; Soloshonok, V. A.; Izawa, K.; Liu, H. Chem. Rev. 2016, 116, 422-518. doi:10.1021/acs.chemrev.5b00392

15. Wang, J.; Sánchez-Roselló, M.; Aceña, J. L.; del Pozo, C.; Sorochinsky, A. E.; Fustero, S.; Soloshonok, V. A.; Liu, H. Chem. Rev. 2014, 114, 2432-2506. doi:10.1021/cr4002879

16. Kirk, K. L. J. Fluorine Chem. 2006, 127, 1013-1029. doi:10.1016/j.jluchem.2006.06.007

17. Bégué, J.-P.; Bonnet-Delpon, D. J. Fluorine Chem. 2006, 127, 992-1012. doi:10.1016/j.jluchem.2006.05.006

18. Isanbor, C.; O'Hagan, D. J. Fluorine Chem. 2006, 127, 303-319. doi:10.1016/j.jfluchem.2006.01.011

19. Allen, A. D.; Fujio, M.; Mohammed, N.; Tidwell, T. T.; Tsuji, Y. J. Org. Chem. 1997, 62, 246-252. doi:10.1021/jo961387k

20. Mitobe, K.; Terashima, K.; Kawasaki-Takasuka, T.; Agou, T.; Kubota, T.; Yamazaki, T. Eur. J. Org. Chem. 2018, 6944-6951. doi:10.1002/ejoc.201801384

21. Kumon, T.; Yoshida, K.; Yamada, S.; Agou, T.; Kubota, T.; Konno, T. Tetrahedron 2019, 75, 3713-3721. doi:10.1016/j.tet.2019.05.042

22. Ghavtadze, N.; Fröhlich, R.; Bergander, K.; Würthwein, E.-U. Synthesis 2008, 3397-3406. doi:10.1055/s-0028-1083188

23. Chinchilla, R.; Nájera, C. Chem. Rev. 2014, 114, 1783-1826. doi:10.1021/cr400133p 
24. Gandeepan, P.; Cheng, C.-H. Acc. Chem. Res. 2015, 48, 1194-1206. doi:10.1021/ar500463r

25. Boyarskiy, V. P.; Ryabukhin, D. S.; Bokach, N. A.; Vasilyev, A. V. Chem. Rev. 2016, 116, 5894-5986. doi:10.1021/acs.chemrev.5b00514

26. Jeon, S. L.; Kim, J. K.; Son, J. B.; Kim, B. T.; Jeong, I. H. Tetrahedron Lett. 2006, 47, 9107-9111. doi:10.1016/j.tetlet.2006.10.088

27. Kumon, T.; Yamada, S.; Agou, T.; Kubota, T.; Konno, T. J. Fluorine Chem. 2018, 213, 11-17. doi:10.1016/j.jfluchem.2018.06.004

28. Ueda, M.; Ueno, T.; Suyama, Y.; Ryu, I. Tetrahedron Lett. 2017, 58, 2972-2974. doi:10.1016/j.tetlet.2017.06.049

29. The lower regioselectivity when using 2-acetylphenylboronic acid instead of 2-formylphenylboronic (2A) still remains unclear. However, a similar tendency for the regioselectivity has been observed in the cobalt-catalyzed [2 + 3] cycloaddition using an unsymmetrical internal alkyne for the construction of indenol derivatives, see [8].

30. Ernst-Russell, M. A.; Chai, C. L. L.; Wardlaw, J. H.; Elix, J. A. J. Nat. Prod. 2000, 63, 129-131. doi:10.1021/np9903245

31. Morrell, A.; Placzek, M.; Parmley, S.; Grella, B.; Antony, S.; Pommier, Y.; Cushman, M. J. Med. Chem. 2007, 50, 4388-4404. doi:10.1021/jm070307+

32. Turek, M.; Szczęsna, D.; Koprowski, M.; Bałczewski, P. Beilstein J. Org. Chem. 2017, 13, 451-494. doi:10.3762/bjoc.13.48

33. It is well-known that $\alpha$-trifluoromethylated carbonyl compounds have a strongly acidic $\alpha$-hydrogen atom due to the highly electron-withdrawing trifluoromethyl and carbonyl groups and are easily enolized. In the present study, it is therefore highly possible that the cis-isomer generated through the catalytic hydrogenation of the indenone 6 isomerized to the much more thermally stable trans-isomer $\mathbf{7}$ via enolization.

34. Cooke, E.; Paradellis, T. C.; Edward, J. T. Can. J. Chem. 1982, 60, 29-34. doi:10.1139/v82-006

35. Barth, W.; Paquette, L. A. J. Org. Chem. 1985, 50, 2438-2443. doi:10.1021/jo00214a008

36. Generally, the signal of the 2-fluoroalkylated indenols 3 appeared at $-56 \mathrm{ppm}$ and that of the 3-fluoroalkylated indenols 4 appeared at -59 ppm.

37. Minatti, A.; Zheng, X.; Buchwald, S. L. J. Org. Chem. 2007, 72, 9253-9258. doi:10.1021/jo701741y

38. Lin, P.-S.; Jeganmohan, M.; Cheng, C.-H. Chem. - Eur. J. 2008, 14, 11296-11299. doi:10.1002/chem.200801858

39. Since a fluoroalkyl group has a very strong electron-withdrawing ability, the Rf-C $\alpha-[\mathrm{Co}]$ bond may be stronger than the $\mathrm{C} \beta-[\mathrm{Co}]$ bond, which stabilizes the transition state of the insertion step. Therefore, Int-2a was produced as the major regioisomeric intermediate.

40. Hamada, Y.; Kawasaki-Takasuka, T.; Yamazaki, T. Beilstein J. Org. Chem. 2017, 13, 1507-1512. doi:10.3762/bjoc.13.149

\section{License and Terms}

This is an Open Access article under the terms of the Creative Commons Attribution License (https://creativecommons.org/licenses/by/4.0). Please note that the reuse, redistribution and reproduction in particular requires that the authors and source are credited.

The license is subject to the Beilstein Journal of Organic Chemistry terms and conditions: (https://www.beilstein-journals.org/bjoc)

The definitive version of this article is the electronic one which can be found at:

https://doi.org/10.3762/bjoc.16.184 